\title{
Le non-verbal dans la relation duelle
}

Jacques Cosnier

\section{OpenEdition \\ Journals}

Édition électronique

URL : https://journals.openedition.org/communicationorganisation/2403

DOI : 10.4000/communicationorganisation.2403

ISSN : $1775-3546$

\section{Éditeur}

Presses universitaires de Bordeaux

\section{Édition imprimée}

Date de publication : 1 novembre 2000

ISSN : 1168-5549

\section{Référence électronique}

Jacques Cosnier, «Le non-verbal dans la relation duelle », Communication et organisation [En ligne], 18| 2000, mis en ligne le 22 janvier 2021, consulté le 05 août 2021. URL : http://journals.openedition.org/ communicationorganisation/2403 ; DOI : https://doi.org/10.4000/communicationorganisation.2403

Ce document a été généré automatiquement le 5 août 2021

(C) Presses universitaires de Bordeaux 


\title{
Le non-verbal dans la relation duelle
}

\author{
Jacques Cosnier
}

\section{Préalables}

1 Deux commentaires préalables me paraissent indispensables : sur la relation duelle d'abord, sur le non-verbal ensuite.

2 La relation duelle peut être de nature extrêmement variée : depuis le corps à corps amoureux jusqu'à la demande de renseignements à l'agent de police, la séance psychanalytique, l'interaction de guichet, la partie de pétanque etc..

3 Ces dernières années cependant le prototype préféré de relation duelle choisi par les chercheurs est la "conversation", situation paradigmatique de base dont on peut faire dériver toutes les autres situations en manipulant ses différents paramètres.

4 La rencontre est un terme encore plus générique souvent utilisé depuis Goffman pour englober tous les genres d'interaction : elle désigne un épisode d'interactions de face-àface (ou plus généralement de "co-présence ») qui commence lorsque des individus reconnaissent leur présence mutuelle et directe, et se termine lorsqu'ils se retirent. Quel qu'en soit le type, l'organisation temporelle générale d'une rencontre comprend 3 temps : ouverture et définition de la situation, déroulement des échanges, clôture.

5 Le premier temps et le dernier sont marqués par des gestes et des paroles rituels: salutations gestuelles et verbales.

6 Le déroulement lui-même peut être plus ou moins ritualisé selon le degré de formalisation de la relation et selon son cadre mais s'appuie sur un dispositif de maintenance: - système de synchronisation des interactants - et système des passages de tours de parole.

7 Quant au non-verbal, je ne reprendrai pas sa définition déjà discutée au cours de la table ronde; en le limitant aux situations conversationnelles habituelles, nous avons vu qu'il comprend aussi bien de l'acoustique (la mise en Voix du Verbal) que du visuel, 
mais je ne traiterai que de ce dernier aspect, sacrifiant à un usage établi, dont il convient pourtant de signaler le biais réducteur.

8 Cependant, même réduit au visuel, j'ai aussi mentionné que le non-verbal comprend plusieurs aspects: des aspects statiques (ou contextuels) et des aspects kinésiques (ou cotextuels).

Des aspects statiques: marques (sexe, âge, ethnie, biotype), marqueurs (vêtements, coiffure, etc.), proxémique (imposée ou choisie).

Des aspects kinésiques : posturo-mimo-gestualité.

11 En fonction de ces différent éléments on peut schématiser les données de base caractéristiques d'une rencontre selon le tableau suivant

Plan d'analyse situationnelle

\begin{tabular}{l|l} 
Cadre & $\begin{array}{l}\text { site-lieu-agencement proxémique } \\
\text { temporalité } \\
\text { finalité et programmes } \\
\text { règles de cadrage } \\
\text { assistance (public éventuel) }\end{array}$
\end{tabular}

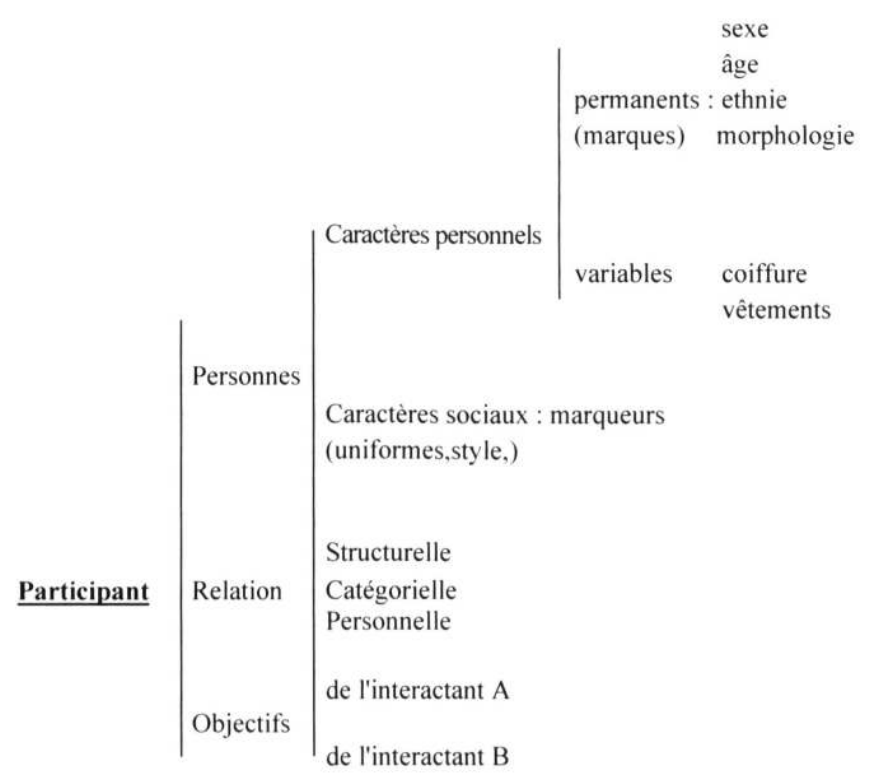

PLATE-FORME COMmUNicative COMmUNe : histoire relationnelle, compétences et savoirs partagés de A et $B$.

12 Ma tâche serait donc d'évaluer la part du non-verbal aussi bien dans les temps de la rencontre que dans ses composants, mais comme il apparaît vite que le non-verbal est pratiquement omniprésent, mon exposé se limitera au champ réduit du non-verbal visuel, kinésique et le plus souvent co-verbal ou conversationnel, adoptant donc le point de vue général d'une relation duelle virtuelle entre des partenaires aussi idéals que le sont les fameux locuteurs de Chomsky. 


\section{Le non-verbal et le contenu propositionnel}

13 Je commencerai par ce qui apparait le plus manifeste dans la situation de rencontre conversationnelle: on échange des tours de parole, or ces paroles s'accompagnent de gestes co-verbaux.

Cette gestualité co-verbale a été très abordée dans les années 1960-1970, sous l'égide du structuralisme triomphant et de la théorie de la communication associés à un intérêt croissant pour le « non-verbal » et la « communication multicanale».

Les différents auteurs qui s'occupèrent alors des gestes avaient le désir de démontrer qu'ils fonctionnaient selon le modèle signifiant/signifié, voire comme Birdwhistell, qu'ils étaient construits de kinèmes et de morphèmes, et ces auteurs aboutissaient à des catégorisations sémiotiques que l'on peut résumer ainsi :

- gestes déictiques (gestes de désignation du réfèrent) gestes illustratifs (du contenu verbal), soit concrets : gestes iconiques. soit abstraits : gestes métaphoriques (ou «idéographiques »).

- bâtons (ou Beats ou Intonatifs), gestes scandant le discours en accord

- avec la construction syntaxique. Cette gestualité étant par définition co-verbale, il s'en suit que son statut est lié directement au contenu propositionnel et à la syntaxe de l'acte illocutoire avec lesquels elle peut être :

- en simple redondance : c'est-à-dire ne faire que répéter par la

- figuration gestuelle ce qui est déjà parfaitement explicité dans le

- discours verbal

- en convergence: elle ajoute par elle-même une information supplémentaire à celle véhiculée par la verbalité; par chaque canal sont produites des informations différentes mais focalisées sur le même objet du discours

- en complémentarité : elle apporte une information indispensable à la compréhension de la production verbale en indépendance : elle véhicule des informations parallèles à celles de la verbalité mais sur un autre objet ou sur un autre aspect de la situation

- en contradiction : elle délivre une information opposée à celle de la chaîne verbale

Deux autres catégories de gestes étaient généralement ajoutées : les quasi-linguistiques (ou emblèmes) gestes conventionnels pouvant fonctionner sans la parole, et les expressifs, essentiellement faciaux, transcatégoriels dans la mesure où ils peuvent être superposés à toutes les catégories précédentes et qui sont devenus l'objet d'une véritable spécialisation à la suite des travaux d'Ekman et de la création du FACS (Facial Action Coding System).

17 Ces expressifs connotent émotionnellement le contenu du discours; ils jouent alors le rôle de qualificatifs, en redondance ou en convergence avec les éléments verbaux concomitants, ils peuvent aussi servir de modalisateurs de l'énoncé verbal en précisant l'attitude affective de l'émetteur vis-à-vis de son propre énoncé selon un procédé souvent utilisé dans l'humour, jouant alors un rôle métacommunicatif parfois décisif pour l'interprétation des paroles énoncées, enfin ils peuvent servir à la régulation interactionnelle comme nous le verrons plus loin.

18 En outre, faut-il ajouter les gestes "extracommunicatifs ", ainsi dénommés car ils ne sont pas reconnus explicitement comme communicants; ce sont d'une part les «adaptateurs» ou activités parasites: grattage, automanipulations, manipulations d'objets etc. aux fonctions réputées autocalmantes, et d'autre part les activités praxiques de travail ou de sport... 
Cette classification fournit une panoplie efficace pour caractériser la gestualité discursive propositionnelle, l'ensemble (gestualité + vocalité + verbalité) réalisant un énoncé total ou " totexte ». Ajoutons que ce totexte n'est pas le résultat d'une addition de signaux plurisémiotiques, mais d'une combinaison (au sens chimique) de ceux-ci.

Cependant si cette classification s'est trouvée validée (aux termes près) par la plupart des chercheurs et a pu satisfaire les sémioticiens, avec les développements de la linguistique et de l'anthropologie interactionnistes son apport réel apparaît aujourd'hui plus complexe que dans l'engouement structuraliste initial : la posturomimo-gestualité sert bien à la constitution du totexte-énoncé, mais en deçà elle sert aussi au travail énonciatif et au delà elle sert à gérer le déroulement de l'interaction.

\section{Le non-verbal et l'interactio}

Si les études contemporaines sur la conversation et ses dérivés ont pris l'essor que l'on connaît, c'est en effet grâce à la prise en compte de l'« interaction ", c'est-à-dire du fait que les énoncés produits au cours d'une rencontre sont co-produits par les locuteurs.

\section{La « synchronie interactionnelle » et lu danse des interactants}

La « synchronie interactionnelle » décrite en 1966 par Condon et Ogston nous retiendra peu malgré son importance historique et terminologique car elle constitue aujourd'hui une notion devenue « classique ».

Par un ingénieux dispositif Condon a analysé image par image des fragments d'interaction filmée. Il a pu ainsi décrire les phénomènes d'autosynchronie et d'hétérosynchronie.

L'autosynchronie : désigne la correspondance chez le locuteur des événements paroliers et des mouvements des divers segments corporels enregistrés.

L'hétérosynchronie: désigne la correspondance chez l'allocutaire d'activités segmentaires synchrones des événements paroliers produits par son partenairelocuteur.

Ces phénomènes réalisent une «danse des interlocuteurs » selon une métaphore très parlante.

27 Avec du recul, malgré leurs imperfections techniques souvent critiquées, ces travaux princeps ont abouti à la formulation de concepts nouveaux et féconds.

Dans des études polygraphiques comprenant entre autres des enregistrements de l'activité phonatoire et de l'activité globale, c'est-à-dire permettant de corréler la présence de la parole et de mouvements nous avons pu constater (Dahan 1969, Economides 1975, Bekdache 1976) :

29 qu'il est vrai que la parole coïncide avec des mouvements chez le parleur dans environ $90 \%$ du temps de prise de parole : on sait qui parle rien qu'à voir celui qui bouge.

qu'il est vrai aussi que le receveur bouge quand l'autre parle, mais à des moments très précis sur lesquels nous reviendrons qu'enfin il existe des différences interindividuelles importantes selon ce que nous avons décrit comme "organisation verbo-viscéromotrice » : certaines personnes étant très «motorisées", d'autres au contraire très « sobres » pour ne pas dire « figées ». 
31 Au total, ces études "gestétiques » (au sens de Pike) convergent pour dire que dans l'interaction, paroles et gestes du parleur sont souvent associés et s'accompagnent à certains moments précis de réactions motrices du receveur. La conclusion sommaire de ces approches micro-descriptives serait « quand ça parle, ça bouge à l'unisson ».

Il faut en fait pour mieux cerner le problème passer au niveau d'étude supérieur, et aborder les données d'ordre gestémique qui nous montrent que la synchronisation interactionnelle est le résultat de deux processus complémentaires: la régulation interactionnelle et l'accordage empathique.

La régulation interactionnelle et le co pilotage de l'interaction

\section{La maintenance des tours et les quatre questions du parleur}

Sous le terme de maintenance nous désignons le processus sous-jacent aux échanges verbaux qui permet à chaque locuteur de gérer au mieux sa participation, c'est-à-dire d'accéder à la «félicité interactionnelle»: pouvoir expliciter sa pensée, la faire comprendre et au-delà être approuvé, partager un point de vue, faire réaliser une action, convaincre etc....

Pour ce, le parleur s'efforce d'être informé sur quatre points, que nous avons appelé les « 4 questions du parleur»: est-ce qu'on m'entend? est-ce qu'on m'écoute ? est-ce qu'on me comprend? qu'est-ce qu'on en pense?

Or, la réponse à ces questions nécessite 1/au minimum un regard du receveur 2/des indices rétroactifs sous la forme d'émissions voco-verbales et/ou kinésiques du receveur.

37 Ce système interactif qui sert à la régulation de l'échange se décompose ainsi en émissions du parleur (activité «phatique»), et en émissions du receveur (activité « régulatrice »).

Du côté phatique, le regard constitue un des éléments majeurs de ce système d'interrégulation et va constituer un «signal intra-tour » selon l'expression de Duncan et Fiske («Speaker within turn signal»). Le parleur en effet, ne regarde pas en permanence le receveur, ce qui donne à son regard valeur de signal. Il l'utilise à certains moments précis de son discours, souvent à un point de complétude vocale et sémantique, de pause brève. Ce signal intra-tour se doit d'être bref pour ne pas être pris pour une proposition de passage de tour, et peut s'appuyer sur un signal gestuel: geste ou maintien de la main dans une position active qui indique que le tour n'est pas fini.

Le signal phatique intra-tour va provoquer les signaux rétroactifs ou régulateurs du receveur («back-channel signal» de Duncan et Fiske) qui peuvent être de plusieurs formes :

- Brèves émissions verbales ou vocales : Hum, oui, d'accord, je vois, non ?, etc.

- Complétudes propositionnelles et reformulations.

- Demandes de clarification : «Comment ça ?.... ». « tu veux dire que ?....»

- Mouvements de tête : très souvent « hochement », singulier ou pluriel.

Mimiques faciales: le sourire en est un exemple fréquent, mais il n'est pas rare d'observer des mimiques de « perplexité » ou de « doute » voire de " réprobation » dont on suppose aisément qu'elles vont influencer la suite discursive du parleur. Le rôle essentiel du regard dans ce système régulateur a été précisé par C. Goodwin (1981) qui en a fait une étude très complète et a souligné son rôle dans l'«organisation 
conversationnelle ». Le parleur a besoin du regard du receveur, et met en œuvre des techniques subtiles pour le provoquer, le regard est utilisé aussi pour marquer l'engagement et le désengagement et ainsi permettre la suspension ou la reprise de la conversation, il l'est aussi pour la désignation de l'allocutaire quand l'interaction se fait à plus de deux personnes.

Enfin, le regard intervient au-delà de l'aspect interactionnel dans l'aspect relationnel, nous y reviendrons.

\section{Les tours de paroles}

Je rappellerai que pour les conversationnalistes. tels Sacks, Schegloff et Jefferson (1974) les échanges conversationnels (dans leur aspect cependant uniquement verbal) sont faits d'une succession de "tours de paroles", dont le chevauchement constitue une violation des règles conversationnelles et oblige l'un des interlocuteurs à s'interrompre rapidement. Pour éviter cette situation critique existent donc des règles de base qui servent à gérer la construction du tour, l'allocation du locuteur suivant, et la coordination du transfert de tour avec le minimum d'intervalle et de chevauchement.

En fait. l'« alternance des tours » n'est pas une règle conventionnelle de nature sociale, mais simplement la conséquence d'une nécessité physiologique: il est facile de démontrer qu'on ne peut pas parler et écouter en même temps. Ce qui est par contre déterminé culturellement, c'est le droit à la parole, et en cas de chevauchement, le droit de la conserver, tandis que dans les cas de situation égalitaire, le «gagnant du tour » s'affirmera le plus souvent en utilisant des procédés non verbaux.

Le parleur proposera le changement de tour en émettant un ensemble d'indices: verbaux (complétude grammaticale, syntagmes conclusifs : voyez-vous, bien...) vocaux (intonation descendante, syllabe prolongée) et kinésiques (regard vers le partenaire, absence de geste illustra tif. éventuellement geste déictique vers l'allocataire désigné).

L'écouteur de son côté peut envoyer des indices de candidature à la parole: détournement du regard, mouvements de tête, raclement de gorge et inspirations préparatoires à la parole, geste de la main à la fois « bâton » et déictique, changement de posture etc....

\section{L'accordage empathique et l'analyseur corporel}

Les notions précédentes, système des tours de parole et procédure de maintenance, nous ont permis de mettre en relief quelques aspects fondamentaux de la participation des gestes à l'interaction. Mais la quatrième question du parleur (« qu'est-ce qu'on en pense » ?) mérite d'être mieux explicitée car elle nous pousse à aborder les problèmes d'empathie et de communication affective, problèmes jusqu'ici peu abordés par les conversationnalistes,

En branchement direct sur les échanges référentiels ou idéationnels et sur les procédures opératoires interactives décrites jusqu'ici, se poursuit dans toute communication interindividuelle un travail sur les affects: travail d'attribution d'affects à autrui et travail d'exposition de ses propres affects.

En situation d'interaction les locuteurs vont gérer leurs propres sentiments, gérer l'expression de ces sentiments réels ou affichés, et s'efforcer de percevoir les 
mouvements analogues en cours chez leur partenaire (ces phénomènes s'effectuent en grande partie de façon non-consciente).

L'échange informationnel et opératoire véhiculé par les tours paroliers et la gestualité co-verbale se doublera en effet d'un échange d'indices et d'indicateurs émotionnels (nous utilisons «indices» pour la communication émotionnelle spontanée, et « indicateurs » pour la communication émotive construite, selon la distinction de Arndt et Janney, 1991).

La participation kinésique y est très importante dans un cas comme dans l'autre.

51 Les mimiques faciales sont considérées comme les supports expressifs privilégiés des diverses émotions, elles en indiqueraient la "qualité ", tandis que les autres indices corporels, gestes, postures révéleraient plutôt l'intensité (Ekman et Friesen, 1967), ou leur tonus (aspect figé du déprimé, expressif de l'excité, sthénique du paranoïaque...).

Un aspect important de l'attribution affective est constitué par le phénomène d'échoïsation ou de synchronie mimétique: les interlocuteurs extériorisent "en miroir " des mimiques, des gestes et des postures semblables. Le sourire et les rires appellent le sourire et les rires, les pleurs, les pleurs ou du moins une mimique compassionnelle etc. Les « mines de circonstance » sont fréquentes, mais de plus, souvent contagieuses.

Ceci nous a amené à deux sortes de considérations.

(mais aussi sans doute en partie le parleur) au-delà du décodage des signaux phatiques et du texte propositionnel qui lui sont destinés, il utilise son propre corps comme source d'information sur ce qui se passe dans le corps de son partenaire par un processus d'échoïsation parfois manifeste, souvent subliminaire. Manifeste : ce sont les convergences d'attitude, les gestes et mimiques en miroir, éventuellement les mouvements labiaux mimétiques...

Cette induction émotionnelle par la reproduction des modèles effecteurs pourrait être mise au service de la connaissance des affects d'autrui. On en relève de nombreux exemples dans les moments d'interaction où les partenaires ont l'impression de bien s'accorder : « d'être sur la même longueur d'ondes » (Cosnier et Brunei, 1994).

Ces activités mimétiques ouvertes ou couvertes, explicites ou subjectives nous ont suggéré le concept « d'analyseur corporel » étayé par plusieurs types d'arguments.

Des données psychophysiologiques telles celles d'Ekman et coll. (1983) : si l'on demande à des sujets de produire telle ou telle expression faciale (on ne lui dit pas de quelle mimique il s'agit, mais on lui dit de contracter tel ensemble de muscles) on constate (1) l'apparition de phénomènes végétatifs caractéristiques de l'émotion (2) des éprouvés subjectifs correspondants (3) éventuellement des fantasmes.

Des données expérimentales mettant en évidence le recours à son propre corps pour évoquer des affects ou pour les reconnaître. Nous avons montré par exemple (avec S. Muyghues-Despointes) dans un expérimentation que nous appelons « les mimiques du créateur » que les sujets à qui l'on demande de dessiner des expressions faciales de la colère, de la joie, de la tristesse, de la surprise, du dégoût... exprimaient facialement eux-mêmes ces émotions et les utilisaient comme modèle proprioceptif.

Dans d'autres expériences, dites « les mimiques de l'interprète » (avec N. Bonnet), nous avons présenté une série de six photos d'une personne entrain de parler, et nous avons demandé à une série de sujets d'inventer les paroles. Ici encore nous avons pu observer les nombreuses échoïsations gestuelles. 
60 Les résultats de ces expériences vont dans le même sens que ceux des nombreuses observations d'échoïsations faciales des sujets à qui l'on demande d'identifier les émotions exprimées sur des dessins ou des photographies (Hess et col. 1998).

Des données neuro-physiologiques: la perception de gestes chez autrui s'accompagne d'activités cérébrales analogues à celles qui apparaîtraient si le sujet observateur accomplissait lui-même le geste (Jeannerod, 1997).

62 Enfin ajoutons que cette conception de l'analyseur corporel, rejoint la théorie motrice de la perception de la parole de Liberman : la reconnaissance de la parole se fait grâce à une reproduction articulatoire automatique et non consciente. Ce processus de reconnaissance cénesthésique des données non-verbales par reproduction mentalomotrice du modèle effecteur serait donc un processus général à la base d'un processus empathico-inférentiel associé au processus cognitivo-inférentiel (l'échange de signaux) déjà signalé.

63 La seconde catégorie de considérations concerne plus particulièrement la gestualité du parleur.

64 Parmi les gestes liés à l'activité énonciative certains sont nécessaires à l'intelligibilité de l'énoncé verbal comme les quasi-linguistiques quand ils sont utilisés comme substituts aux mots, de même certains déictiques et parfois certains illustratifs. Mais 11 faut reconnaître que l'énoncé est le plus souvent intelligible même en l'absence de la plupart des illustratifs co-verbaux (d'ailleurs on peut remarquer que les présentateurs de télévision sont souvent cadrés avec la tête et le haut du tronc sans que leurs membres soient visibles). Ces illustratifs seraient donc à première vue plus utiles pour le parleur que pour le receveur et c'est un fait que l'inhibition des co-verbaux. ou leur contrainte externe, nuit au flot associatif : la figuration corporelle (pensée imagée de D. McNeill) facilite la mise en mots de la pensée et lui fournit une assise indispensable.

65 Mais au-delà de cette facilitation, si l'on admet la théorie de l'analyseur corporel, la mise en scène corporelle de la pensée du parleur facilite aussi le processus empathicoinférentiel du receveur, la gestualité énonciative est un inducteur d'échoïsation : si l'énonciateur pense et parle avec son corps, l'énonciataire perçoit et interprète aussi avec son corps.

66 Ce dispositif à la fois cognitivo et empathico-inférentiel varie selon la relation des partenaires de la rencontre: symétrie vs complémentarité, sympathie vs antipathie, selon les structures caractérielles de chacun (distanciation obsessionnelle vs rapproché et identification hystérique). Il serait un des éléments fondamentaux de la "convergence communicative " (expression positive de l'« engagement», de l'«affiliation» ou de l'«intimité») caractérisée par: le sourire et les mimiques syntones, le contact oculaire, l'orientation frontale du tronc, l'inclinaison antérieure, les hochements de tête, la gesticulation co-verbale, l'ensemble portant au maximum la synchronie interactionnelle, tandis qu'à l'opposé la « divergence » serait marquée par l'asynchronie des mimiques et l'absence de sourire, la fréquence des extracommunicatifs autocentrés, l'inclinaison postérieure, les mouvements des jambes et l'immobilité des bras, la rareté des hochements de tête et autres régulateurs. (Mehrabian, 1971).

De tout cela ressort que, comme le répétait Goffman, toute rencontre est un jeu subtil dans lequel chacun s'engage et s'expose à tel point que des règles protectrices des faces sont omniprésentes sous la forme de rituels, de routines et de partitions « ready- 
made » qui font que généralement ça ne marche pas trop mal et même que beaucoup aiment ça.

\section{L'accommodation et l'homéostasie relationnelle}

Dans les paragraphes précédents nous avons examiné les événements moteurs cotextuels et leur participation à la gestion de l'interaction. Nous ajouterons quelques mots sur d'autres éléments non verbaux, "attitudes" posturales, intensité et amplitude des gestes et des mimiques qui associés aux marques physiques et aux marqueurs socio-culturels créent un "climat» contextuel. Certains de ces éléments font partie du « décor » et restent permanents au cours de la rencontre, mais d'autres traduisent l'accommodation situationnelle et c'est eux qui nous intéressent ici, en particulier les indicateurs de relation et les paramètres kinésiques du contrôle social.

Par contrôle social (Patterson, 1976) on désigne le processus mis en œuvre pour réaliser une action finalisée ou/et pour influencer les réactions d'autrui dans un sens déterminé. On quitte donc ici la situation égalitaire et informelle de l'interaction conversationnelle pour aborder les situations asymétriques, telles les interactions de sites qui obéissent à des scripts préalablement définis avec des distributions de rôles contraignantes mais aussi les interactions faussement conversationnelles: repas d'affaires, diverses situations de séduction, de persuasion etc....

Dans ces situations de contrôle social, on retrouvera bien sûr les différents éléments de base décrits précédemment mais qui ici seront modalisés en fonction des statuts, de la dominance et des objectifs explicites ou cryptiques de la relation. Ils apparaitront donc au cours du déroulement de la rencontre en fonction de l'organisation temporelle et des conditions contextuelles.

71 Ainsi peut-on observer les techniques très codifiées de prise de contact et d'ouverture de l'interaction avec divers modes d'adresses verbales et d'échanges gestuels, mimiques et tactiles : baisers, poignées de main, accolades selon la catégorie de partenaires et les statuts réciproques, etc.

Durant la période de déroulement de la rencontre le regard joue un rôle majeur dans la différenciation des statuts dominant-dominé (Thayer. 1969; Exline. 1971). Dans les interactions ordinaires homme-homme, le fait de porter des regards prolongés est jugé plus dominant que des regards rares ou furtifs. C'est l'asymétrie de l'utilisation des regards, fréquence et durée qui est significative.

73 Le toucher constitue aussi un signe indicateur spécial, qui peut manifester (a) l'intimité de la relation (b) mais aussi l'emprise et la dominance et dans ce cas n'est pas réciproque (Henley, 1973); il est initié plus souvent par les hommes que par les femmes, par les plus âgés que par les plus jeunes, par les socio-économiquement plus nantis. Il en est sensiblement de même pour les sourcils froncés et la bouche non souriante (Keating et al., 1977).

74 Cependant plusieurs de ces indicateurs de dominance ont plus une fonction de « rappel » que de conquête : ils confirment un statut déjà établi par d'autres moyens ou inhérent à la situation («reminders » de Summerhayes et Suchner, 1978), ils peuvent aussi servir d'« affiche » et assurer deux fonctions destinées au public éventuel : affiche de relation servant à l'ostention de l'intimité aux tiers (par exemple exagération du rapprocher, des rires, du contact), affiche d'opinion, servant à exprimer à l'attention de 
tiers éventuels l'approbation ou la désapprobation des propos émis par le partenaire (par exemple, en cas d'approbation : des hochements de la tête amples et répétitifs avec le regard non posé sur le parleur). C'est le métalangage de la relation.

Ces diverses accommodations liées au contrôle social seront aussi dépendantes de ce que l'on pourrait appeler l'homéostasie de la relation: maintien d'un équilibre adéquat, c'est-à-dire supportable sinon confortable entre les deux tendances contradictoires, approche et évitement, mises en jeu dans tout rapport interindividuel.

Argyle et Dean en avait fourni en 1965 un modèle dit de "l'équilibre de l'intimité" (Intimacy-equilibrium mode!) : les forces qui poussent un partenaire vers l'autre ou l'en écartent tendent à maintenir un état d'équilibre. Si cet équilibre est perturbé par une intimité trop grande dans une dimension, par exemple des regards trop appuyés, il se rétablit par une compensation sur une autre dimension, par exemple une augmentation de la distance interindividuelle. Un détournement du regard quand l'autre fixe trop longtemps est aussi un moyen fréquent de maintenir l'équilibre.

77 Mais la restauration de l'équilibre peut aussi se faire par un changement de position (retrait du buste, ou rapprochement) et au niveau du canal verbal par un éventuel changement de thème. Ainsi plus la distance interpersonnelle est faible, plus le contact oculaire diminue (Argyle et Dean, 1965; Goldberg. Kiesler et Collins, 1969) et moins l'orientation du corps est directe (Mehrabian et Diamond, 1971; Patterson et Sechret, 1970).

78 Ces modèles sont intéressants dans la mesure où ils montrent la synergie entre les différents paramètres de l'activité énonciative, et la recherche d'un équilibre consensuel à la fois compatible avec l'état affectif propre à chaque interactant, la régulation des échanges en cours et les accommodations aux contraintes contextuelles. Mais ces dernières restent déterminantes pour l'interprétation des phénomènes observés.

\section{En conclusion}

79 Je conclurai ce schématique survol par quelques remarques qui pourraient orienter de futures recherches.

80 Le champ du «non-verbal » même limité à la relation duelle est à la fois très vaste et très hétérogène. En se limitant à ses aspects visuels on peut distinguer des éléments cotextuels liés à l'activité énonciative et à la composition de l'énoncé et des éléments contextuels liés au cadre de l'interaction.

81 Les éléments cotextuels eux-mêmes ont des fonctions multiples. La plus classique est leur contribution à la composition de l'énoncé, mais plusieurs arguments montrent qu'ils participent souvent pour le parleur à l'activité énonciative dans laquelle ils jouent un rôle cognitif et facilitateur (« on ne peut parler sans bouger »).

Par ailleurs le non-verbal co-textuel est essentiel pour la régulation et la coordination de l'interaction, et ce de deux façons complémentaires : par l'utilisation d'un dispositif de signaux culturellement modalisé. et par le processus d'empathie inférentielle qui met en jeu des phénomènes d'échanges corporels plus profonds et moins contrôlables.

La mise en évidence de procédés de cadrage affectif aux côtés des procédés de cadrages cognitifs, ainsi que les notions d'accommodation et de contrôle social ouvrent des 
pistes intéressantes en permettant de mieux situer la participation des éléments non verbaux dans le déroulement des interactions.

Enfin sur le plan pratique, reconnaître l'importance du système de régulation-pilotage et des éléments non-verbaux devrait avoir quelques conséquences :

- En pédagogie où l'on a déjà souvent souligné combien le savoir enseigné nécessite de savoir enseigner.

- De même en formation et en psychothérapie où la pragmatique de la relation est un moteur essentiel de l'évolution et s'accomplit très largement par les canaux vocaux et kinésiques bien souvent à l'insu des protagonistes (voir à ce sujet les étonnants travaux de V. HaynalReymond et de M. Heller).

- Dans les relations interculturelles, car chaque culture ayant dans ce domaine ses propres prescriptions et proscriptions les malentendus-malvus y trouvent un terrain des plus propices à leur éclosion.

- Dans la vie quotidienne enfin dont la mise en scène et les représentations qui s'y jouent donnent souvent autant à voir qu'à entendre.

\section{BIBLIOGRAPHIE}

ARGYLE M..Bodily communication, London, Methuen, 1988.

ARGYLE M., DEAN J., « Eye-contact, distance and affiliation » in Sociometry.

28, pp. 289-304, 1975.

ARGYLE M., SALTER V., NICHOLSON H., WILLIAMS M., BURGESS L., « The communication of inferior and superior attitudes by verbal and non-verbal signals » in British journal of social and clinical psychology, 9. pp. 222-231, 1970.

ARNDT H., JANNEY R.W. « Verbal, prosodie, and kinesic emotive contrasts in speech » in Journal of pragmatics, pp. 522-550. 1991.

BEKDACHE K., L 'organisation verbo-viscéro-motrice au cours de la communication verbale selon la structure spatiale ou proxémique, Thèse $3^{\mathrm{e}}$ cycle, Université Lyon 1, 1976.

CALBRIS G., Geste et motivation. Semiotica 65-l\2, pp. 57-96, 1987. Condon W.S., Ogston W.D., «Sound film analysis of normal and pathological behavior patterns » in Journ. of Nervous and Mental Disease. 143, pp. 338-347, 1966.

COSNIER J., Brossard A., (eds), La communication non-verbale, Delachaux et Niestlé, 1984.

COSNIER J., « Expression et régulation des émotions dans les interactions de la vie quotidienne », Colloque La psychologie sociale et les émotions, Paris, MSH. 5-9 janvier 1987. Cosnier J., Grands tours et petits tours, 1988.

COSNIER J... GELAS N., KERBRAT ORECCHIONI C, Echanges sur la conversation. Paris, Edition du CNRS.

COSNIER J., « Les tours et le co pilotage dans les interactions conversationnelles », 1989. CASTEL R., COSNIER J., JOSEPH I.,Le purler frais d'Erving Goffman, pp. 233-244, Paris, Edition de Minuit. 
Cosnier J, Psychologie des sentiments et des émotions. Paris, Retz, 1994 COSNIER J., « Synchronisation et co pilotage de l'interaction conversationnelle », Protée, 20, 2, pp. 33-39, 1992.

COSNIER J., VAYSSE J., « La fonction référentielle de la kinésique », Protée, 20,2, pp. 40-47, 1992.

COSNIER J.,BRUNEI ML, « De l'interactionnel à l'intersubjectif » in MARCARINO A. (ed). Analisi delle conversazione e prospettive di ricerca in etnometodologia, Urbino. Quattro Venti, pp. 151-163. 1997.

UAHAN G., Contribution au traitement du contexte psychophysiologique de l'examen psychologique. Thèse $3^{\mathrm{e}}$ cycle, Université Lyon 1, 1969. DUNCAN S., FISKE P.W.. Face to face interaction research, Hillsdale. 1977. Economides S., Situations due/les et corrélations psychophysiologiques. Thèse $3^{\mathrm{e}}$ cycle. Université Lyon 1, 1975.

EDINGER J.A., PATTERSON L.M., Non-verbal involvement and social control. Psychological Bulletin, 93. 1, pp. 30-56, 1985.

EKMAN P., Emotion and the human face. Cambridge, Cambridge University Press, ed. 1982.

EKMAN P., FRIESEN W.V., « The repertoire of non-verbal behavior » in Semiotica, 1, pp. 49-98, 1967.

EKMAN P., LEVENSON R., FRIESEN W... « Autonomic nervous system activity distinguishes between emotions » in Science, 221, pp. 1208-1210. 1983. EXLINE R.V., «Visual interaction: the glances of power and preference » in COLE J. K. (ed) Nebraska symposium on motivation (vol.19). Lincoln, University of Nebraska Press, 1971.

GOLDBERG G.N., KIESLER C.A., COLLINS B.E., « Visual behavior and face to face distance during interaction » in Sociometry, 32, pp. 43-53. 1969.

GOLDBERG S., ROSENTHAL R... « Self touching behavior in the job interview » in Journal of non-verbal behavior, 10, pp. 65-80, 1986.

GOODWIN C, Conversational organization, London, Academic Press, 1981.

HALLE M., Stevens K-N., « Speech recognition » in Mehler et Noizet (eds).

Textes pour une psycholinguistique. Mouton, La Haye, 1974.

Haynal-Raymond V. « Manifestations des émotions chez les patients suicidaires » in Plantin (ed) Les émotions dans les interactions, Lyon, PUL, 2000.

HELLER M. « Dynamiques expressives in Plantin » (ed) Les émotions dans les interactions, Lyon, PUL, 2000.

FLENLEY N.M., Body politics: power, sex, and non-verbal communication, Englewood Cliffs, ErenticeHall, 1977.

HESS U.,Blairy S.,Philippot P., « Facial reactions to emotional facial expression » in Cognition and emotion. 12. pp. 509-532, 1998.

HOCHSCHILD A.R., « Emotion work, feeling rules and social structures » in American Journal of Sociology, 85, 3, pp. 551-575. 1979.

JEANNEROD M. « Mental imagery in the motor context » in Neuropsychologic! 33, pp. 1419-1432, 1994. 
KEATING C.F., MAZUR A., SEGALL M.H., « Facial gestures which inflence the perception of status » in Sociometry, 40, pp. 374-378. 1977.

KERBRAT-ORECCHIONI C., Les interactions verbales, Paris, Armand Colin, 1991. Liberman A.M.. Mattingly I.G.. « The motor theory of speech perception revised » in Cognition. 21, pp. 1-36, 1985. MC DOWALL J.J., « Interactional synchrony: a reappraisal » in Journal, of personnality and social Psychology, 36. pp. 936-975, 1978. Mehrabian A., Non-verbal communication, Chicago, Aldine, 1971. Mehrabian A., Diamond S.G., «Verbal and non-verbal interaction of strangers in a waiting situation » in Journal of experiment. Reasearch in Personality, 5, pp. 127-138. 1971.

PATTERSON M.L.. « An arousal model of interpersonal intimacy » in Psychological Review, 83, pp. 235-245. 1976.

SACHS H., SCHEGLOFF E.A.. JEFFERSON G.A., « A simplest systematics for the organization of turntaking for conversation » in Language. 50, pp. 696-735, 1974.

THAYER S., « The effect of interpersonal looking duration on dominance judgments » in Journal of social Psychology, 79, pp. 285-286, 1969. Vaysse J. « La sémiotique des gestes centrés sur le corps et leurs implications langagières dans le site médical » in Semiotica, 91, pp. 319-340, 1988. 